\title{
Raman and Surface-Enhanced Raman Spectroscopy of Fatty Acids and Lipids
}

\author{
Eric Amankwa \\ Humanities and Science, Grand Canyon University, Arizona-Phoenix, United States
}

Email address:

amankwaeric@yahoo.com

\section{To cite this article:}

Eric Amankwa. Raman and Surface-Enhanced Raman Spectroscopy of Fatty Acids and Lipids. Optics. Vol. 10, No. 1, 2021 , pp. 7-22. doi: $10.11648 /$ j.optics.20211001.12

Received: May 4, 2021; Accepted: May 20, 2021; Published: May 27, 2021

\begin{abstract}
The goal of this thesis was to study, determine, and measure Raman and surface-enhanced Raman spectroscopy (SERS) of fatty acids and lipids. Firstly, the Raman measurement was done using silver substrate where the activation process was achieved by focusing crystals of green laser radiation $5 \mathrm{~mW}$ power at 5 minutes on the silver substrate. The Raman measurement again was done using Invia Raman Spectroscopy with $514 \mathrm{~nm}$ excitation and objective 100x magnification where the samples to be measured were incubated using RH6G (good signal analyzer). After the incubation process, the samples were rinsed with water and allowed to dry for 5 minutes where ten samples of fatty acids and lipids were measured, recorded, saved and baseline of the spectra's were corrected using matlab codes and averaged. Secondly the SERS measurement was done by growing silver chloride nanoparticle on the silver substrate where the substrate was dipped in a precursor solution of silver nitrate and sodium chloride in a cyclic process. The photosensitive silver chloride crystals were reduced into silver nanoparticles using laser light from the Invia Raman spectroscopy. The SERS measurement was done by depositing the fatty acids and lipids to be measured on the spot which contains the silver nanoparticle recorded the values, saved and baseline of the spectra's corrected using MatLab codes and averaged. This thesis work reveals that, the peaks obtained by the Raman and SERS measurement originated from the double bonds which was used to identify saturated and unsaturated fatty acids and lipids from one another. The study reveals that, the Raman measurement occurs at higher concentrations whereas the SERS measurement occurs at lower concentrations. The study reveals that the SERS measurement depends on the nature of the analyte, integration time, shape, size and laser power whereas the Raman measurement depends on the surface area and laser power. Lastly, the study reveals that the $514 \mathrm{~nm}$ excitation was negligible to efficiently execute the surface Plasmons of the SERS measurement.
\end{abstract}

Keywords: Spectroscopy, Raman Spectroscopy, Surface Enhanced Raman Spectroscopy, Plasmonic, Substrate

\section{Introduction}

Fatty acid and lipids biology

The identification and investigation of lipids and fatty acids started in the 17th, 18th, 19th, and 20th centuries where researchers used non-optical methods to investigate various structure of lipids and fatty acids. In this article, the following fatty acids and glycerides were studied and characterized using Raman and Surface- Enhanced Raman Spectroscopy (SERS).

16:1 $\mathrm{FA}=$ palmitoleic acid eli cis-9-hexadecenoic acid (omega-7)

16:0 FA = palmitic acid eli hexadecanoic acid

18:1 FA = oleic acid eli cis-9-octadecenoic acid (omega-9)
18:0 FA = stearic acid eli octadecanoic acid

18:2 MAG = sn-1 (sn-3) -linoleic acid MAG

18:0 MAG $=$ sn-1 (sn-3) -stearic acid MAG

18:1/18:0 PC = sn-3-phosphatidylcholine-sn-2-oleic acidsn-1-stearic acid glycerol

22:6/18:0 PC = sn-3-phosphatidylcholine-sn-2-

docosahexanoic acid-sn-1-stearic acid glycerol.

The motivation of this thesis is to identify the spectra ranges of the samples where the peaks originated from, biological difference, various bond position (length), chemical point and measuring point. Again, the library of different lipids and fatty acids spectra's will be kept and compared with spectra of other constituents. In this section below, the uses, features and analyses of fatty acids and lipids are introduced. 


\subsection{Lipids}

Lipids are naturally-occurring compounds named from the Greek word lipos, which means 'fat' are defined as biomolecules or heterogeneous group of compounds that are insoluble in water but soluble in organic solvents [16]. Lipids are organic compounds found both in plants and animals. They contain the following elements hydrogen, carbon and oxygen. Lipids are structurally indispensable to metabolism, high solubility in non-polar solvents, exhibit large amount of energy and low solubility in water. They are also are nonpolar (hydrophobic) compounds soluble only in organic solvents such as chloroform, ether, benzene and acetone. Lipids with membranes are amphipathic, having non-polar end and polar end.

\subsection{Lipids Interaction}

Lipids intermolecular interactions are characterized by hydrophobic effect and van der Waals interactions. Research has shown that, many lipids are amphipathic molecules, interact with other molecules and with water solvents through electrostatic interactions and hydrogen bonding [16].

\subsection{Polar and Non-polar Structure of Lipid}

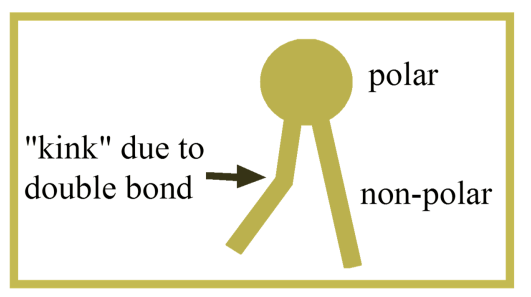

Figure 1. Diagram of polar and non-polar lipid.

Figure 1. Reviews that polar regions of lipids can form considerable interactions with aqueous molecules while the non-polar regions tend to aggregate with other non-polar molecules.

\subsection{Structure of Lipid}

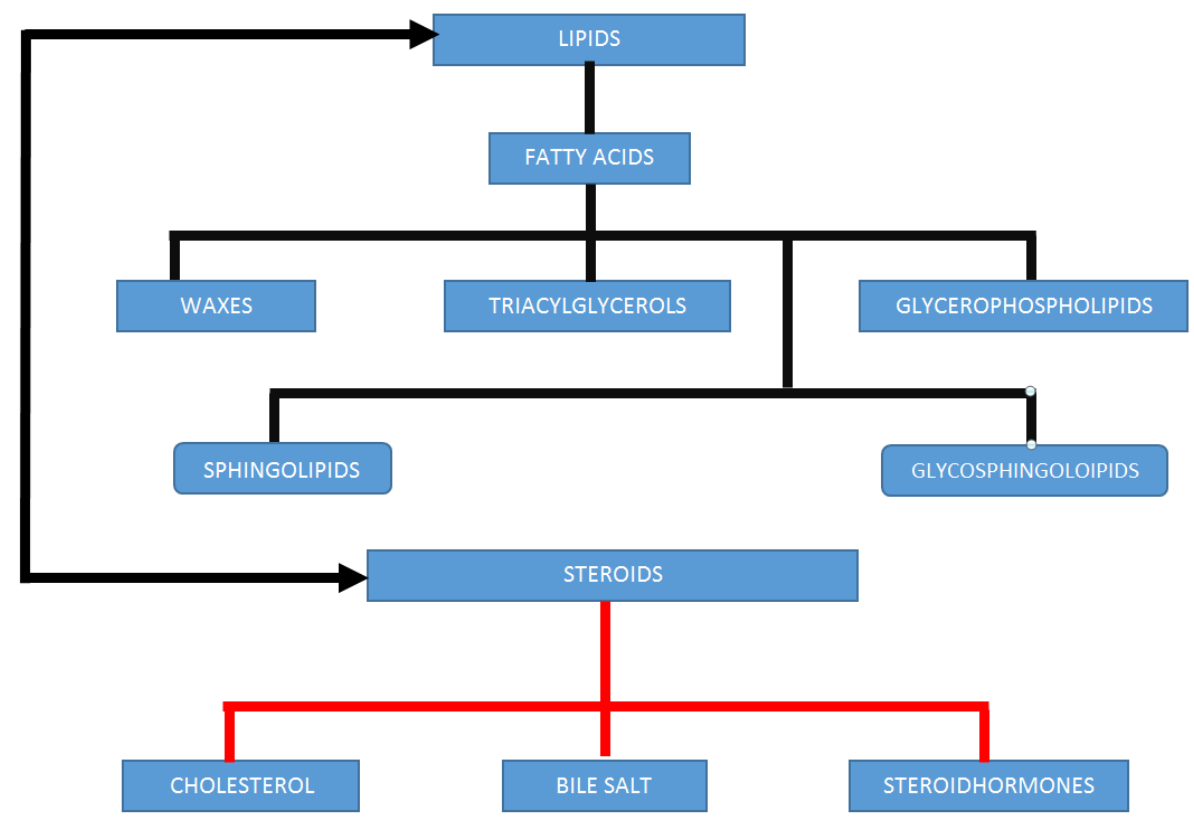

Figure 2. Schematic diagram of lipid.

Figure 2. Reviews that lipids consists indirectly of fatty acids, waxes, triacylglycerols (fats and oils), glycerophospholipids, sphingolipids, glycosphingolipids and directly of steroids.

Figure 2. Also reviews that the indirectly linked lipids contains fatty acids whereas the directly linked lipids (steroids) contains no fatty acids.

\subsection{Factors Affecting Absorption of Lipids and Relevance}

The factors that affect the rate of lipids absorption include: chain length of fatty acids, degree of saturation of fatty acids, overheating and autoxidation, emulsifying agents, and age of subject. Lipids have many benefits to living organisms and these may include the following; forming the structural components of cell membranes, thermal insulation, energy storage molecules, electron carriers, hormonal regulation, enzymatic cofactors, chemical signaling, metabolic fuels and protective coating of plants and animals.

\subsection{Fatty Acids (FA)}

Fatty acids are carboxylic acids (organic acids) that consist specifically of 12 and 20 carbon atoms. They have an even number of carbon atoms because they are built from 2-carbon molecules with long aliphatic tails (long chains) and are unbranched [17].

Fatty acids are normally determined by the saturation and 
unsaturation of the carbon chains. They are insoluble in water due to the fact that the size of the non-polar portion is larger than the size of the polar portion (carboxyl area).

Structure of Fatty Acid

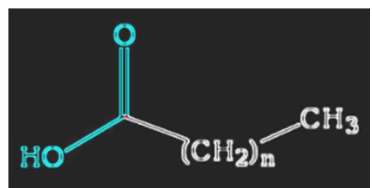

Figure 3. Structure of fatty acid

Figure 3. The tail in white signifies the hydrocarbons whereas the light blue section signifies the carboxylic acids.

\subsubsection{Saturated Fatty Acids (SFA)}

These are fatty acids with a single bond between the carbons

They are solid at room temperature, linear with no bends or kinks, closely packed together in a parallel arrangement, higher London dispersion forces, and higher melting point due to higher attraction between chains.

Illustration of SFA

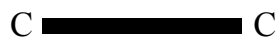

The above illustration reviews that saturated fatty acids (SFA) consist only of a single between the carbon chains.

\subsubsection{Unsaturated Fatty Acids (USFA)}

These are fatty acids with one or more double bonds between the carbon chains. They can be classified as monounsaturated (MUSFA), polyunsaturated (PUSFA), cis or trans. They are also liquid at room temperature, have bends or kinks due to double bonds (cis), have irregular shape due to the molecules, have lower melting point due to fewer attraction between carbon chains, have lower London dispersion forces, and they are not closely packed together in a parallel arrangement rather randomly packed.

\subsubsection{Illustration of USFA}

$\mathrm{C}$ C

The above illustration reviews that unsaturated fatty acids consists of one or more double bonds between the carbon chains.

\subsubsection{Cis Fatty Acids}

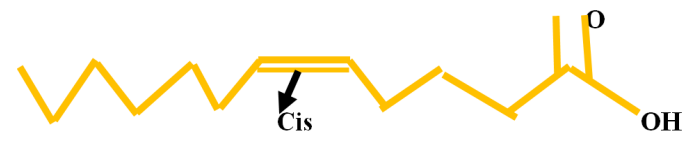

Figure 4. Cis fatty acids.

Figure 4. Reviews unsaturated Cis fatty acid (UCFA) with bulky groups on the same side of the double carbon bond.

\subsubsection{Trans Fatty Acids}

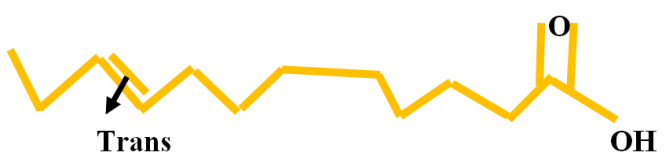

Figure 5. Trans fatty acids.

Figure 5. Reviews unsaturated trans fatty acid (UTFA) with bulk groups on the opposite side of the double carbon bond.

\subsubsection{Diagrammatic Description of Fatty Acids and Lipids Under Study}

Table 1. Shows the description of the samples under study.

\begin{tabular}{|c|c|c|}
\hline SIMPLE NAME & SCIENTIFIC NAME & DESCRIPTION \\
\hline $\begin{array}{l}\text { LLGLa TAG (Linoleic/linoleic/gamma-Linoleic } \\
\text { acid) Triglyceride }\end{array}$ & 6,9,12-Octadecatrienoic acid (Omega-6) & $\begin{array}{l}\text { Liquid at room temperature due to evaporation of } \\
\text { the solvent }\end{array}$ \\
\hline 16:0 FA (Palmitic acid) & Hexadecanoic acid & Solid due to vibrations in the bonds \\
\hline 16:1 FA (Palmitoleic acid) & 9-Hexadecenoic acid (Omega-7) & Amorphous \\
\hline Gla FA (gamma linoleic acid) & 6,9,12-Octadecatrienoic acid (Omega-6) & Amorphous \\
\hline 18:2 MAG (Linoleic acid monoglyceride) & 9,12-Octadecadienoic acid & Amorphous \\
\hline Ala FA (Alpha Linoleic acid) & $9,12,15$-Octadecatrienoic acid & Amorphous \\
\hline $\begin{array}{l}\text { 22:6/18:0 (stearic acid glycerol) phosphatidyl } \\
\text { choline }\end{array}$ & 4,7,10,13,16,19-Docosahexanoic acid (Omega 3) & Amorphous and solid (two phase and two chain) \\
\hline
\end{tabular}

This session describes the various optical methods used for the research.

\subsection{Spectroscopy}

Spectroscopy is the study of the principles of light -matter interaction or the science of analysing and interpreting spectra. Generally, spectroscopy is a term used to describe the interactions of various types of electromagnetic radiation with matter and it involves the analysis of light over a range of wavelengths such as the wavelength of visible light [18].
In spectroscopy electromagnetic wave incident on a sample characterize light after sample and characterize change in sample. The sample is characterized depending on the amount of light absorbed or emitted, or the optical rotation. Absorption is the change in intensity of incident light (transmission) at different frequency or wavelength. Emission includes fluorescence, phosphorescence or Raman scattering and optical rotation is the change of light incident on the sample (rotation of polarization) [3].

The main instrument used in spectroscopy is the 
spectrometer. A spectrometer is a device that is used to measure the properties of light emitted by a source or spectrometer is an instrument used to analyse the nature of light emitted by various sources depending on properties such as refraction, absorption, reflection and interference. The main idea behind the spectrometer is to split light into its components' wavelength, generate a spectrum and determines its physical and chemical properties of materials [1].

The principle behind the operation of spectrometer is that light diffracts as it traverses from one homogeneous medium to another and the diffraction angle is a function of wavelength or frequency. Spectrometers are normally designed to measure specific wavelength range of the electromagnetic spectrum. The choice of components for the design depends on the source of light to be measured. Based on these unique features of spectrometers, they are employed in many fields of study. For example, in astronomy or astrophysics, spectrometers are used to measure velocities of galactic components, composition of stars and Nebula chemical makeup [1].

There are a number of different spectrometers, each of which is designed to achieve different goals but their operational mechanisms are similar. Every spectrometer consists of three major components, diffraction layer made of diffraction grating/glass prism, collimating and focusing lenses/mirrors and detector [21].

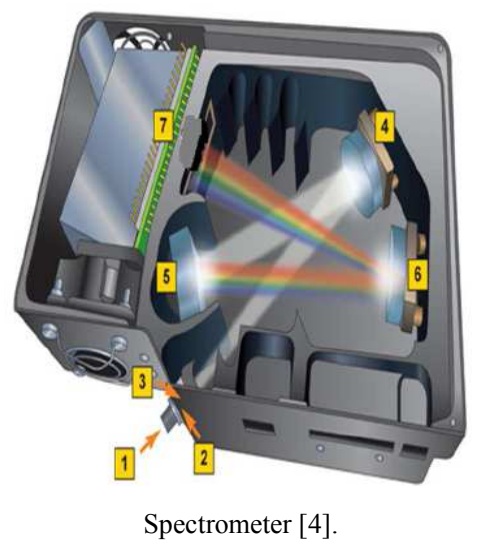

Figure 6. Schematic diagram of how light propagates through a commercial.

Figure 6. describe the general working principle of a spectrometer. Light from a given source enters from position (1) through fixed entrance slits (2 and 3) which specify the width of the incident beam. The thin beam of light travels the length of the housing until it reaches a lens/mirror (4), which collimates the beam. The collimated beam is then intersected by diffraction grating (5) which disperses the different wavelengths (colours) of light, because the diffraction angle is a function of wavelength. This dispersed beam is collected and focused on the detector (7) by the focusing mirror/lens (6). The properties of the light is then measured based on the scales on the detector. Most detectors [8] are calibrated to measure the intensity or polarization state of the light beam [10].

Theory of Raman and Surface-Enhanced Raman Spectroscopy (SERS)

\subsubsection{Raman Spectroscopy}

Raman spectroscopy started in 1920Fs which explains the inelastic scattering of photons by molecule [19]. In Raman spectroscopy, the molecule either gains or loses its energy based on the vibrational or rotational states of the molecules where the first is called (anti-stokes) and the last (stokes) [7].

Rayleigh scattering is another phenomenon where the photons scattered retains their normal or original energy and just negligible amount is Raman scattered.

In the field of spectroscopy, Raman spectroscopy competes with other non-optical methods such as mass spectroscopy and gas chromatography (MS-GC) where the production of signals failed and good results had not been achieved due to poor enhancement where vibrational and rotational states of molecules were invisible until the research conducted by Raman managed to overcome this trail [14].

Another example is the initial observation of pyridine Raman spectra on roughed silver in 1974 [9] was discovered and researchers could not tell where the Raman peaks originated from until a new discovery in 1977 [11] where researchers begun to show interest with the use of surface enhanced Raman spectroscopy (SERS).

Raman research could not yield good results due to low excitation (weak scatterer) thus minimal view concentration analyte and appearance of fluorescence anytime the incident light moves to blue until researchers came out with SurfaceEnhanced Raman spectroscopy (SERS) where this required an enhancement no matter the amount of light source applied. The enhancement administered could be either particles or metal surfaces [12].

In quantum physics, energy is always quantized due to the vibrational states of the molecules, polarizability and the bonds between them. There is always inelastic and elastic impact between the molecules due to a change in the electromagnetic field of the molecules.

In practice the Raman measurement is done by using laser light (monochromatic) to excite the internal vibrational modes of the molecules to its virtual state of excitation where spectrometer is used to collect and analyse the scattered light as shown in Figure 7. [24].

The amount of scattered light is very negligible compared to the excitation where a narrow wavelength band laser filter light is used to segregate the various wavelength from the original wavelength as shown in Figure 7. [20].

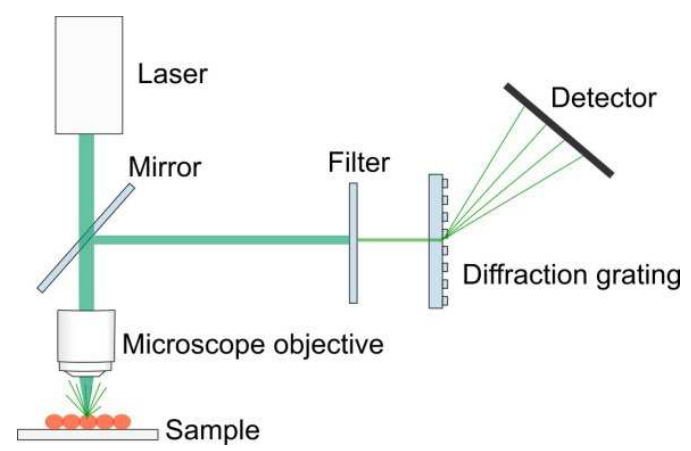

Figure 7. Raman Measurement principle. 
The international standardized unit for the Raman shift is $1 / \mathrm{cm}$.

The transformation in the shift frequency (wavelength) of the light is called the Raman Scattering and this depends upon the vibrational states of the molecules where the Raman shifted photons could be either lower or higher energy and the molecular system, the wavelengths could be associated with vibrational, rotational, electronic level transition and polarization states of the wavelengths.

Raman is applicable in fields such as pharmaceuticals, material science and forensic investigations.

\subsubsection{Surface Enhanced Raman Spectroscopy (SERS)}

There is co-existence between SERS and plasmons. Plasmon is a quantum of plasma oscillations [22] while SERS relies on the electromagnetic enhancement principle [3].

Electromagnetic enhancement occurs due to the exciting light that interacts with the electrons on the surface to form plasmons. This Plasmon is the localised surface plasmons (LSPs) in which the analyte is adsorbed on a surface, usually a rough surface or a chosen surface, where the excitation frequency will excite the Plasmon and causes scattering [2].

The Plasmon energy is then transferred to the adsorbed molecules where the Raman process usually occurs on the molecule and negligible amount of energy is observed due to the surface scattering and difference in the varying shifted wavelength light [10].

The enhancement factor is directly proportional to the electromagnetic field to the fourth power [13] where different excitations could be observed in gaps, crevices, pits, tips, clefts and hot spots where the magnitude of the electromagnetic field is very high.

The enhancement factor for SERS is within $10^{10}-10^{11}$ due to occurrence of the electromagnetic field [5]. SERS is applicable in, DNA hybridization [6], in vitro and in vivo medical diagnostics [15].

\subsection{Plasmonic Substances}

The progress of SERS depends on the amount of substance or material used. The most common materials used for SERS are silver $(\mathrm{Ag})$ and gold $(\mathrm{Au})$ where both have localised surface polariton resonance (LSPR) that extend to all the visible and near infrared regions in which most of the Raman measurements are observed. This silver (Ag) and gold (Au) nanostructure will be explained in the substrate preparation thus methodology section.

In practice the analyte to be measured is deposited on a SERS substrate which could be either gold or silver. After illumination with laser light, the metallic structure interacts with the light and the localised surface plasmons are then excited due to the concentration of the light at the vicinity of the metal surface [2].

The hotspot areas (highly concentrated) then enhance the intensity of the Raman Scattering originating from the molecules as shown in Figure 8.

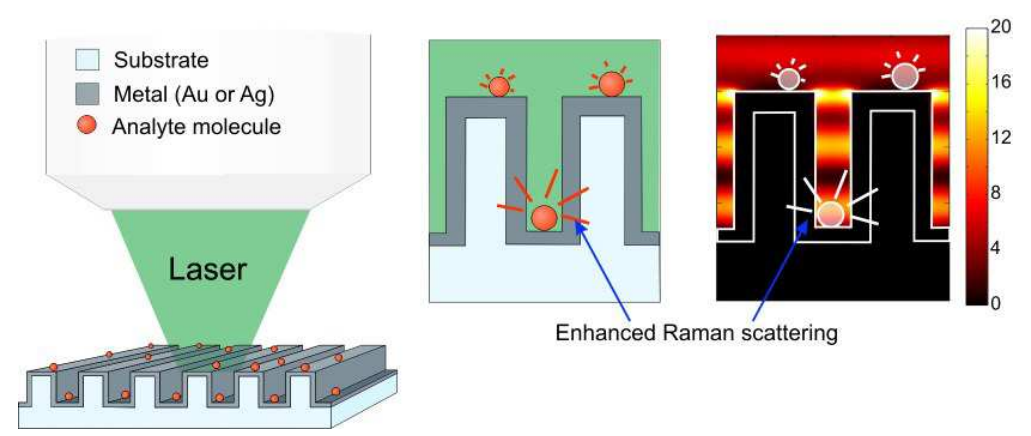

Figure 8. SERS measurement principle (Pasi et al., 2016).

\subsection{Solubility Values at Room Temperature (SVRT)}

Table 2. Solubility Values.

\begin{tabular}{lll}
\hline FORMULA & SUBSTANCE & SVRT \\
\hline $\mathrm{AgNO}_{3}$ & silver nitrate & 216 \\
$\mathrm{NaCl}$ & sodium chloride & 35.89 \\
$\mathrm{AgCl}$ & silver chloride & $1.923 \times 10^{-4}$ \\
$\mathrm{NaNO}_{3}$ & sodium nitrate & 87.6 \\
\hline
\end{tabular}

Table 2: reviews that the highest solubility substance was silver nitrate (216), followed by sodium nitrate (87.6), sodium chloride (35.89) and silver chloride which is very negligible soluble in sub milli molar range at $\left(1.923 \times 10^{-4}\right)$.

Table 2 again reviews that the solubility values determine the variations in concentration which depicts that silver nitrate has a higher concentration and is directly proportional with the solubility value at room temperature in increasing order of magnitude whereas sodium nitrate, sodium chloride and silver chloride follow respectively.

The higher the solubility value the higher concentration means that the solubility value depends greatly on the concentration.

\section{Objectives}

The aim of the thesis work is to research the trends in biophotonics where specific fatty acids and lipids were studied to identify their spectra ranges whether they are within monosaturated (MS), polyunsaturated (PUS) or saturated (S) also where the peaks originated from.

Secondly, the thesis aims to find out which optical technique or spectroscopic method is relevant thus Raman or 
SERS compare their peaks and similarities and also the substrate preparation for the characterization.

Lastly, it will target to identify biological difference, various bond length, chemical point and measuring point of the various fatty acids and lipids under study.

\section{Methodology: Substrate Growth Preparation}

\subsection{Solvent Preparation}

2 part of methanol and 1 part of chloroform were used in the preparation where $5 \mathrm{mg}$ of fatty acids and glycerides each were dissolved in $500 \mu \mathrm{l}$ of MetOH: chloroform 2:1 with fatty acid content of $10 \mathrm{mg} / \mathrm{ml}$.

\subsection{Dipping and Silver Chloride Growth Process (Fabrication)}

The silver chloride coating occurred by placing a magnetic stirrer in both precursors for uniformity in concentration with the substrate placed on the suction cup of the dipping machine as shown in Figure 9.

The substrate to be coated was submerged repeatedly in a precursor solution of silver nitrate and sodium chloride in a cyclic manner by setting the programme software for 50 cycles of substrate dipping process at ambient temperature as shown Figure 9 with the applicable dipping parameters in table 3 at a final concentration range in measurement of
$50 \mathrm{mM}-150 \mathrm{mM}$ at ratio $1: 100$.

This yields nucleation of ions thus silver (positively ions) and chloride (negatively ions) growing silver chloride $(\mathrm{AgCl})$ crystals on the surface of the substrate based on the dipping parameters in Table 2 and yields the equation:

$$
\mathrm{AgNO}_{3}(\mathrm{aq})+\mathrm{NaCl}(\mathrm{aq})-->\mathrm{AgCl}(\mathrm{s})+\mathrm{NaNO}_{3}(\mathrm{aq})(1)
$$

Silver chloride itself is not SERS-active until the crystals is turn into metallic silver through photoreduction thus metallic silver nanostructures as shown in the operational process Figure 9

The chosen substrate considered in this thesis work is silicon substrate due to the following reasons;

1. The silicon substrate can be used in conventional planar SERS.

2. The crystals can be imaged non-invasively using scanning electron microscope (SEM) less conductive coating.

\subsection{Robot Dipping Parameters}

Table 3. 50 cycles of dipping parameters.

\begin{tabular}{llll}
\hline Position/step & Soak time (sec) & Up delay (sec) & Set up \\
\hline 1 & 1.0 & 0.1 & $1: 360$ \\
2 & 0.1 & 0.1 & $2: 608$ \\
3 & 1.0 & 0.5 & $3: 824$ \\
4 & 0.1 & 0.5 & $4: 600$ \\
\hline
\end{tabular}

\subsection{Pictorial View of the Dipping Process}

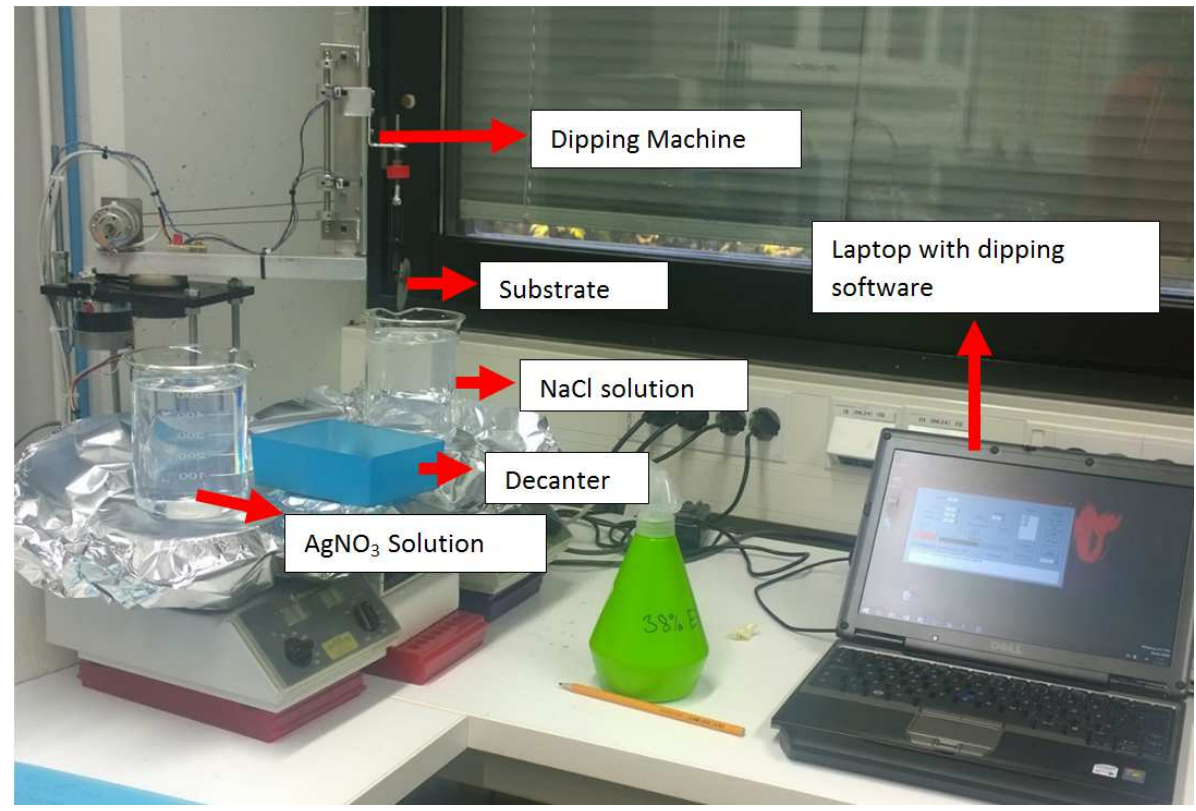

Figure 9. Diagram showing the operational view of the dipping machine.

\subsection{Practical Measurements (Raman and Sers)}

\subsubsection{Raman Measurement}

Invia Raman Microscope with $514 \mathrm{~nm}$ green laser excitation, Numerical aperture (NA) of 0.4 and objective
(Magnification) 100x were the parameters used.

The activation process was done by focusing crystals of green laser radiation at power $5 \mathrm{mw}$ at 5 minutes and micro diameter spot of 20 . 
The samples to be measured were incubated using RH6G (one micro molar) because it is a good Raman Scatter, gives good signal and excellent analyzer for 10 minutes as well as water rinsed and allowed to dry.

The real Raman measurement were measured and recorded for ten samples of fatty acids and lipids at various locations as shown in Figure 7 based on the following parameters exposure time, power and spot diameter respectively as 10 seconds, 60 microwatts and 5 micrometers with corrected baseline using matlab.

\subsubsection{Pictorial View of the Raman Spectroscopy}

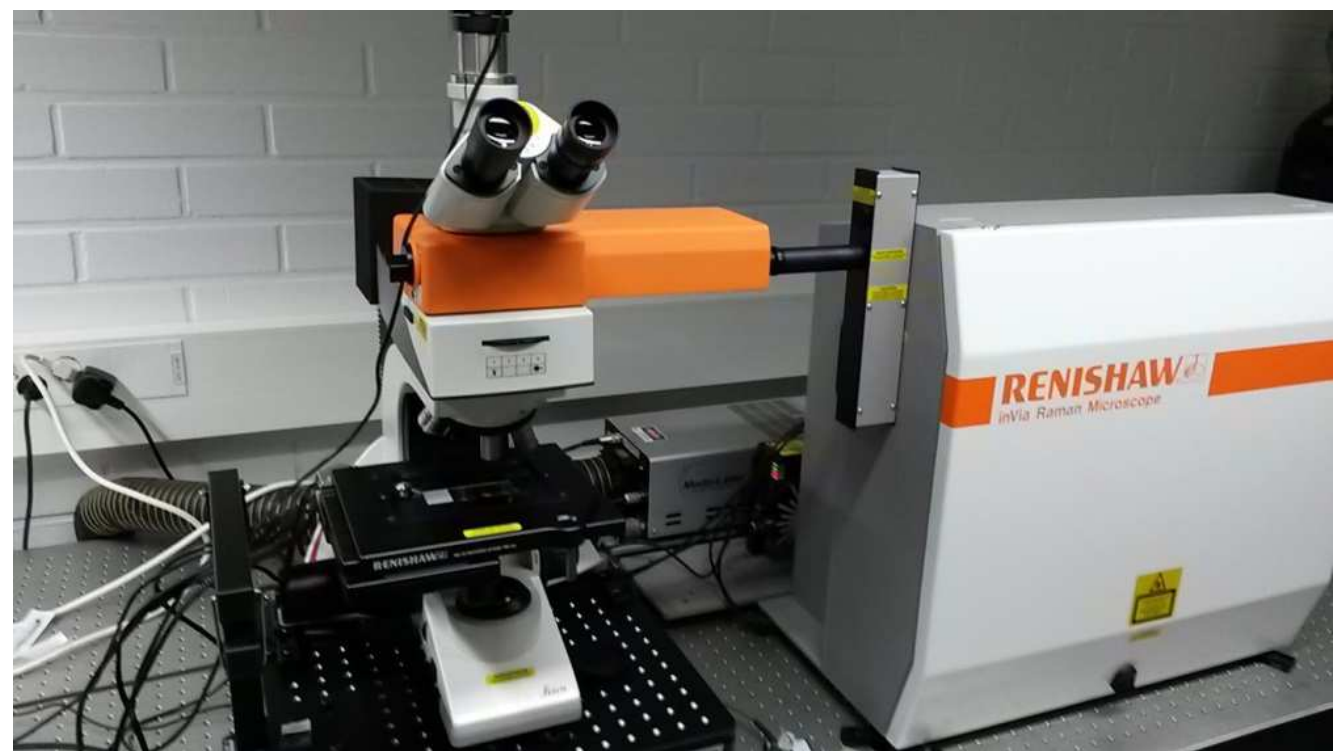

Figure 10. Showing real View of the Invia Raman spectroscopy.

\subsubsection{SERS Measurement}

The silver chloride substrate is not SERS-active and has to be reduced into metallic silver nanoparticle to receive the correct signals required for the SERS and the plasmonic features. Activation (hot spot) was done by photo-reducing the substrate with green laser of the Raman Microscope as shown in Figure 11 based on the following parameters laser wavelength, power and duration exposure of the laser respectively as $514 \mathrm{~nm}, 5 \mathrm{~mW}$ and 2 minutes.

Firstly, the enhancement was achieved by deposition of
Rhodamine $6 \mathrm{G}$ on the silver chloride substrate and initial measurement done and recorded because Rh6G gives good signal enhancement and excellent analyzer. The actual SERS measurement was done and recorded for five different samples of fatty acids and lipids as shown in figure 11 based on the following parameters green laser wavelength, power, varying time and Magnification (Objective) respectively as $514 \mathrm{~nm}, 50$ micro watt, 10 seconds and 100x. Lastly the average baseline for the measured and recorded samples was corrected using matlab.

\subsubsection{SERS Measurement Set up}

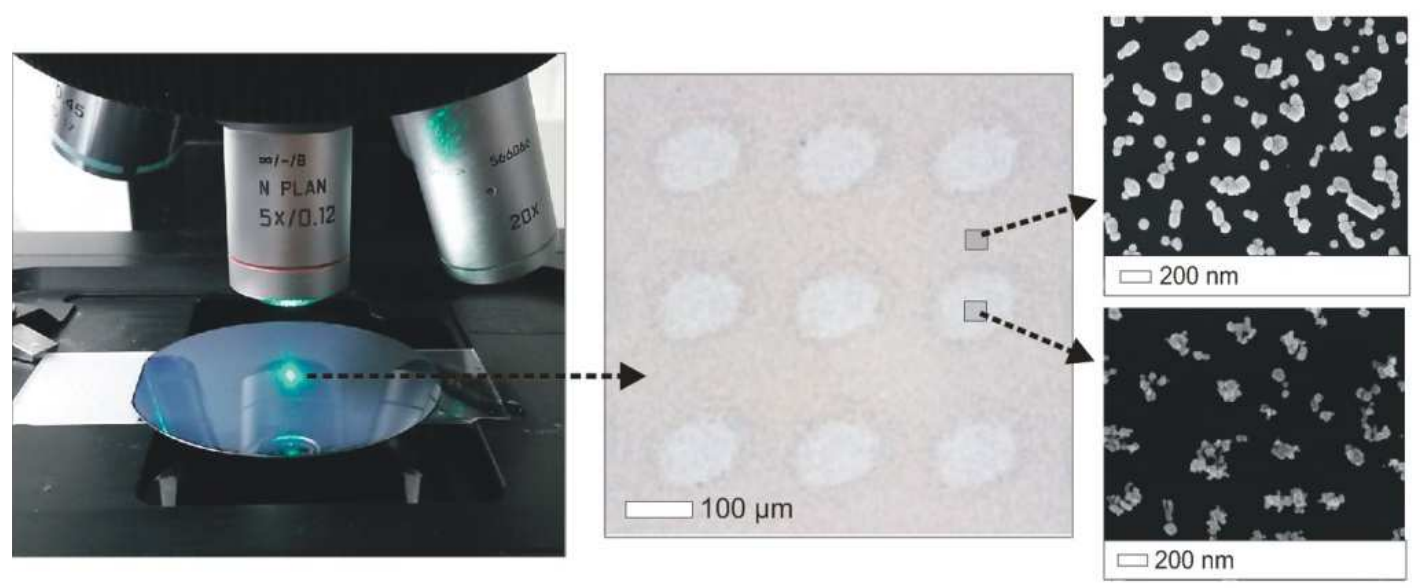

Figure 11. Showing the SERS measurement set up (Pasi et al., 2016). 


\subsubsection{Results of the Raman Measurement (RM)}
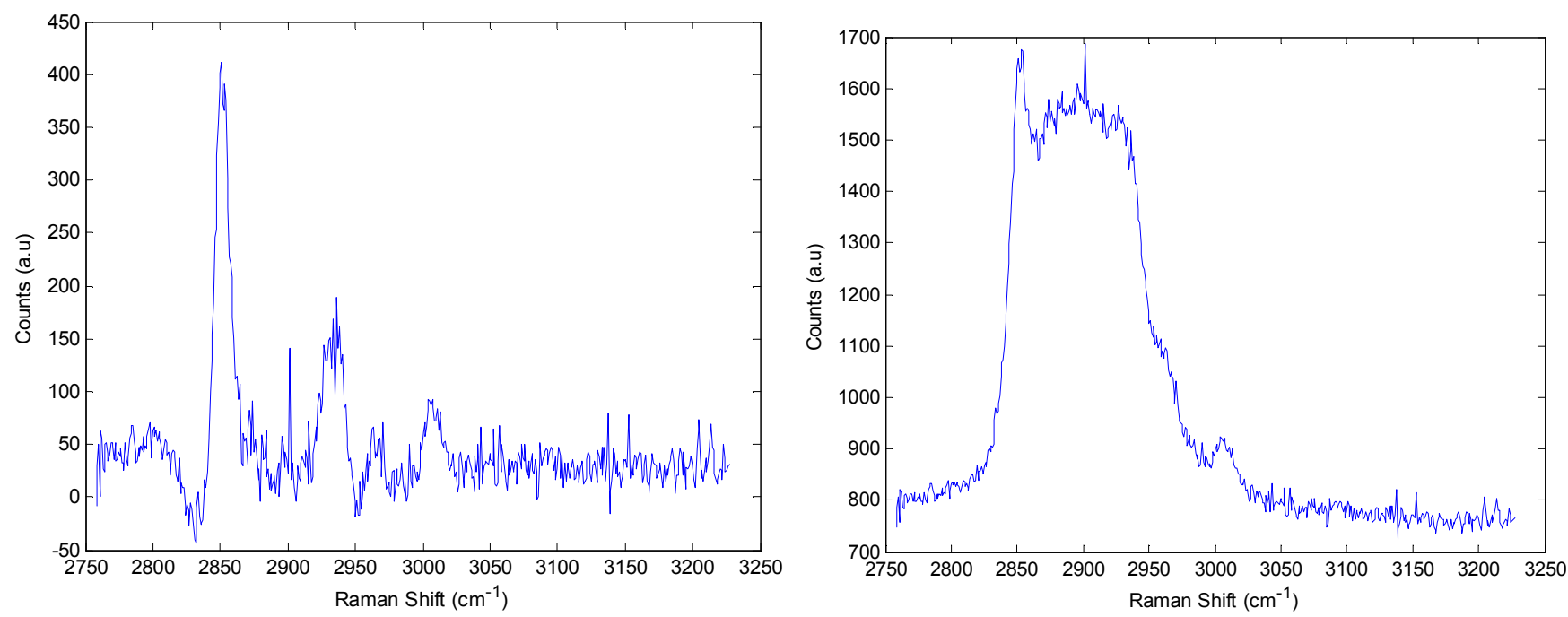

Figure 12. Stearic acid eli octadecanoic acid (Fatty acid 18:0).
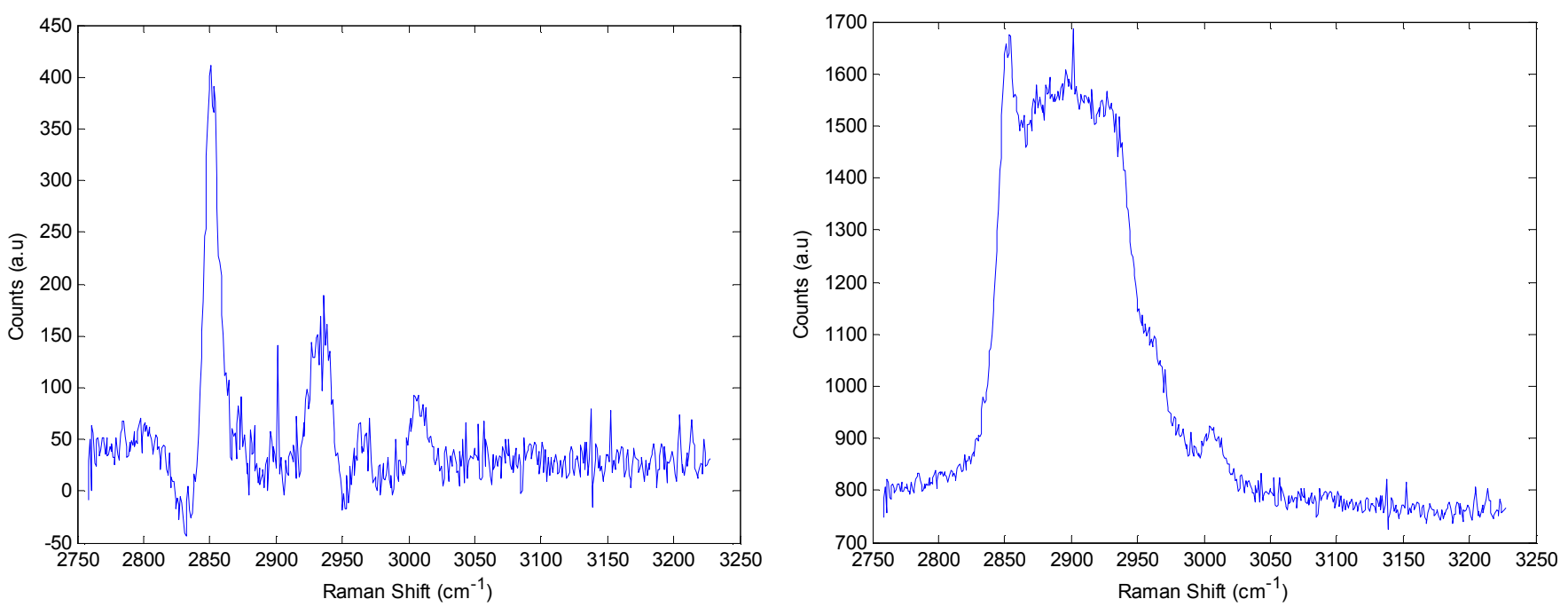

Figure 13. Oleic acid eli cis -9-octadecanoic acid omega-9 (Fatty acid 18:1).
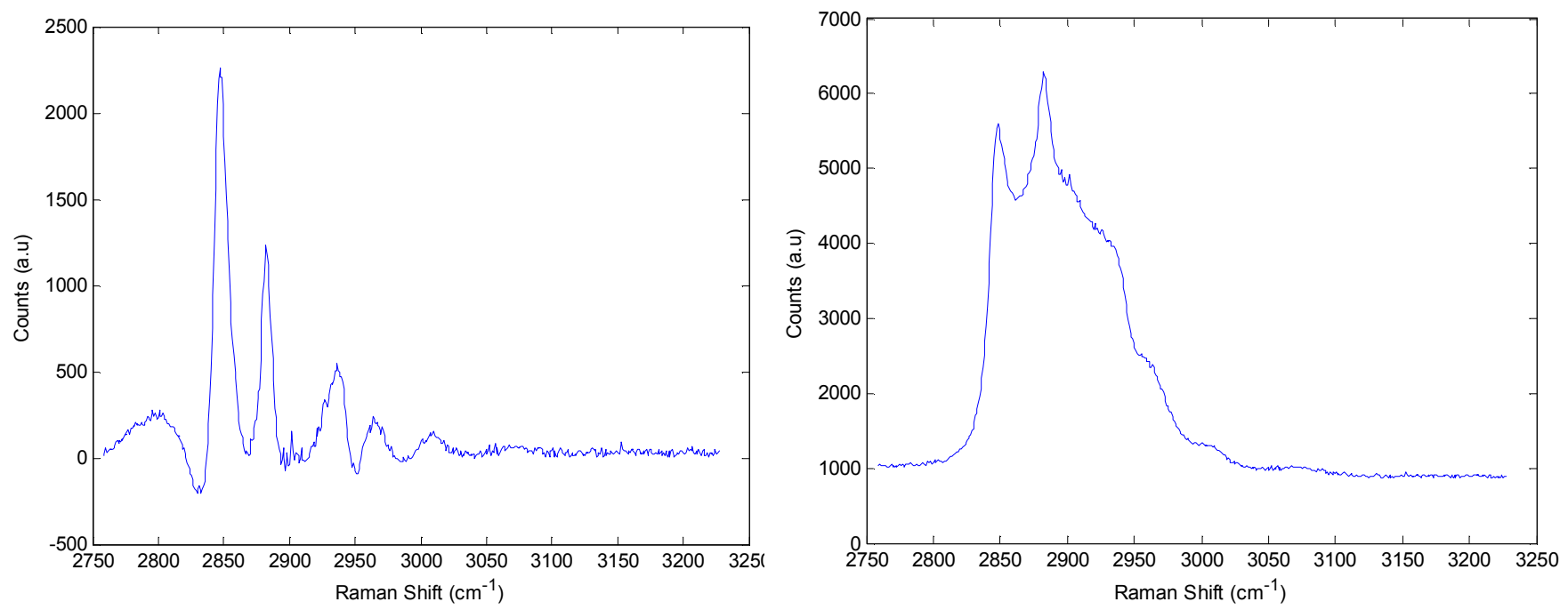

Figure 14. sn-3 (sn-3)-stearic acid Monoglyceride (MAG 18:0). 

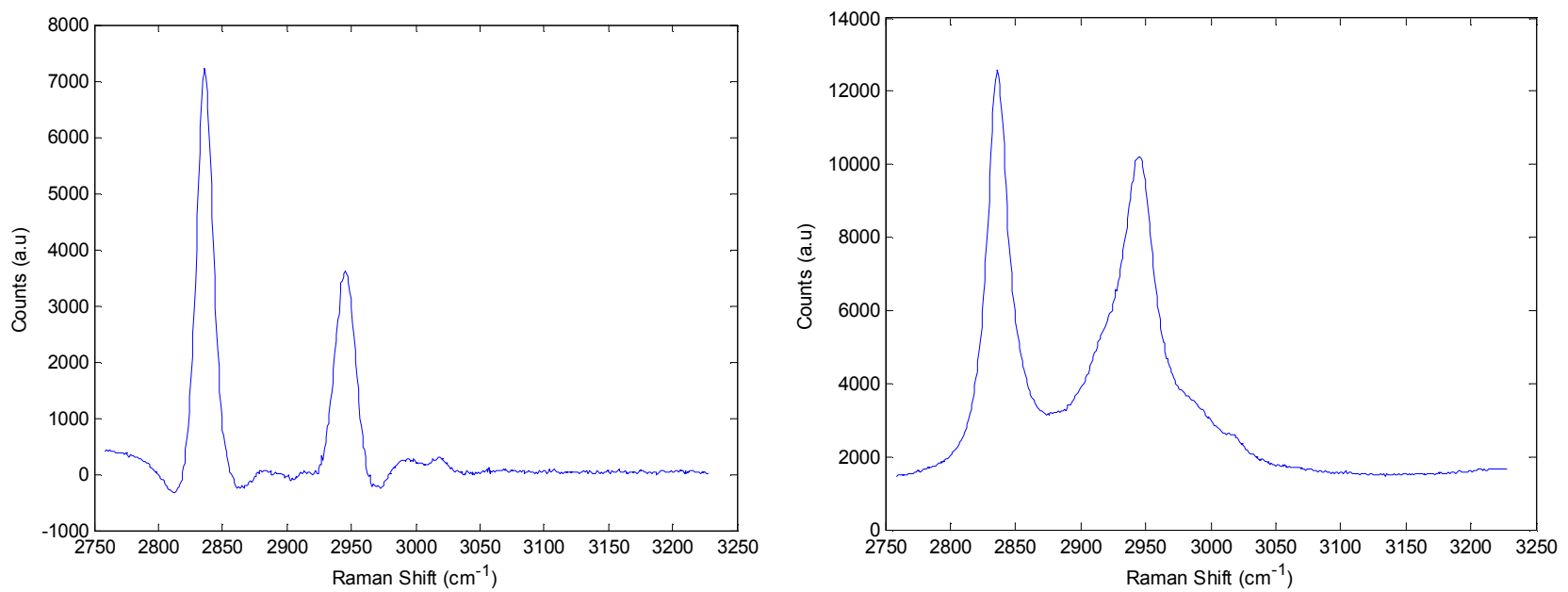

Figure 15. Palmitic acid eli hexadecanoic acid (Fatty acid 16:0).
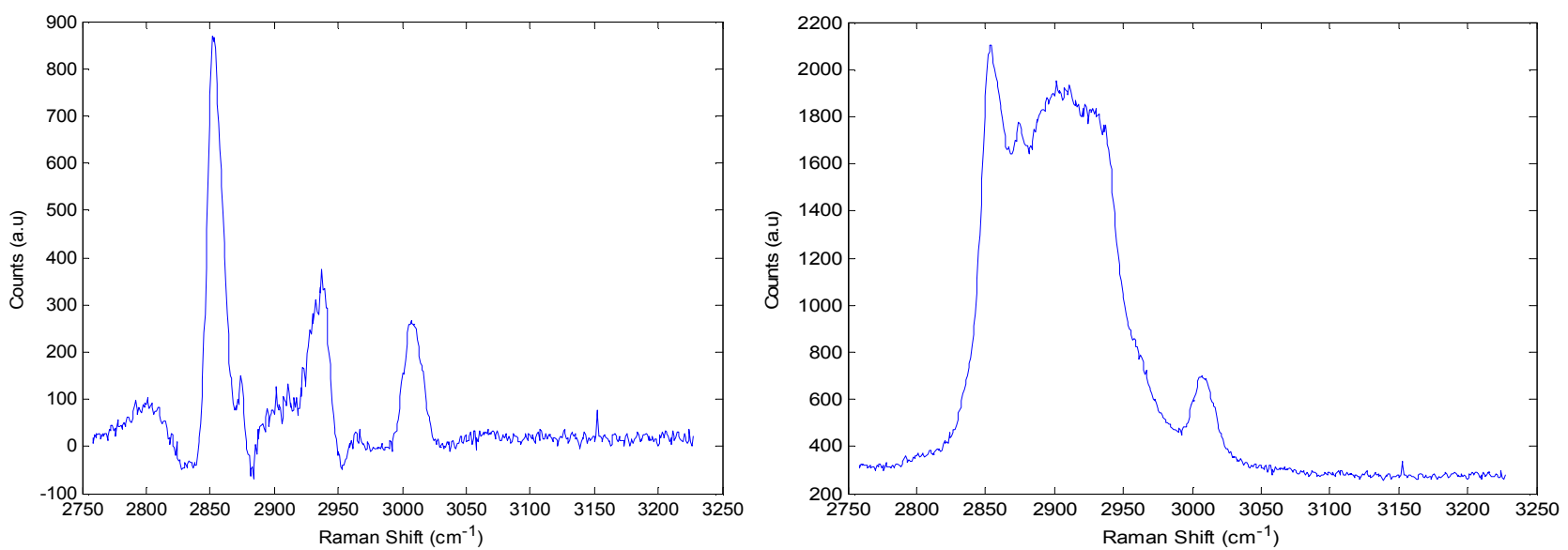

Figure 16. Palmitoleic acid eli cis 9-hexadecenoic acid (Omega-7 Fatty acid 16:1).
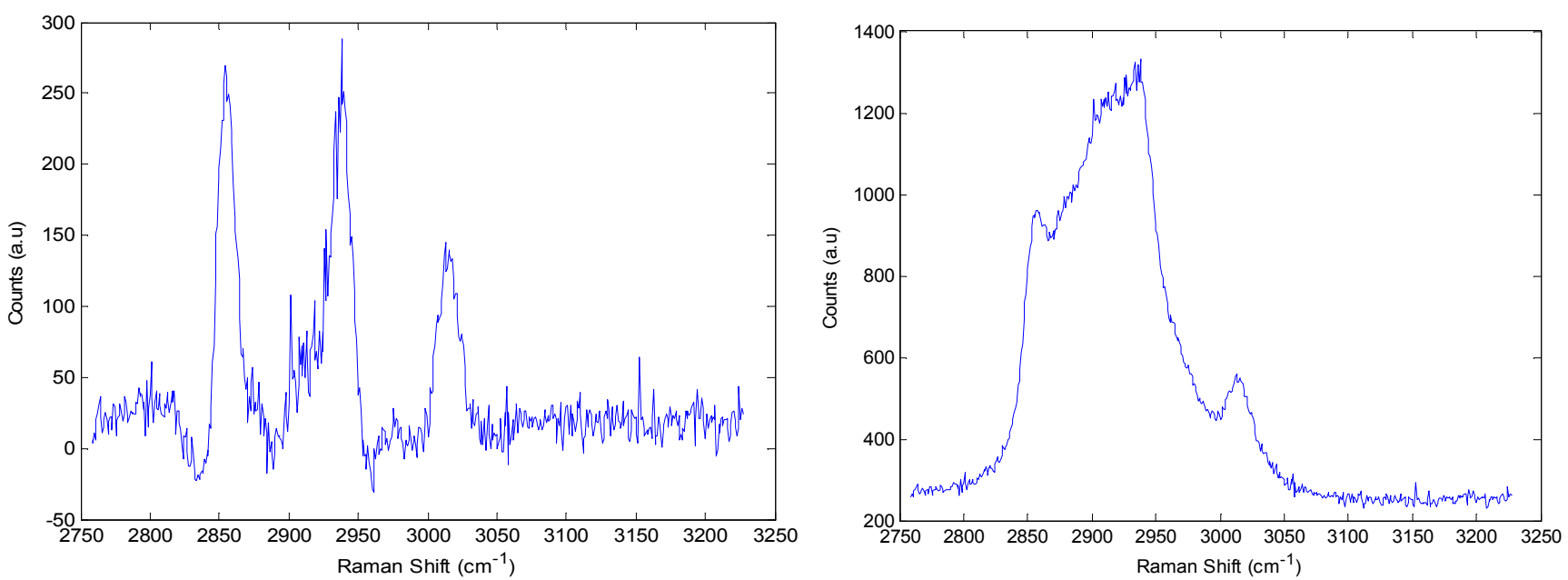

Figure 17. Alpha-linolenic acid eli all-cis-9, 12, 15-octadecatrienoic acid (Omega-3). 

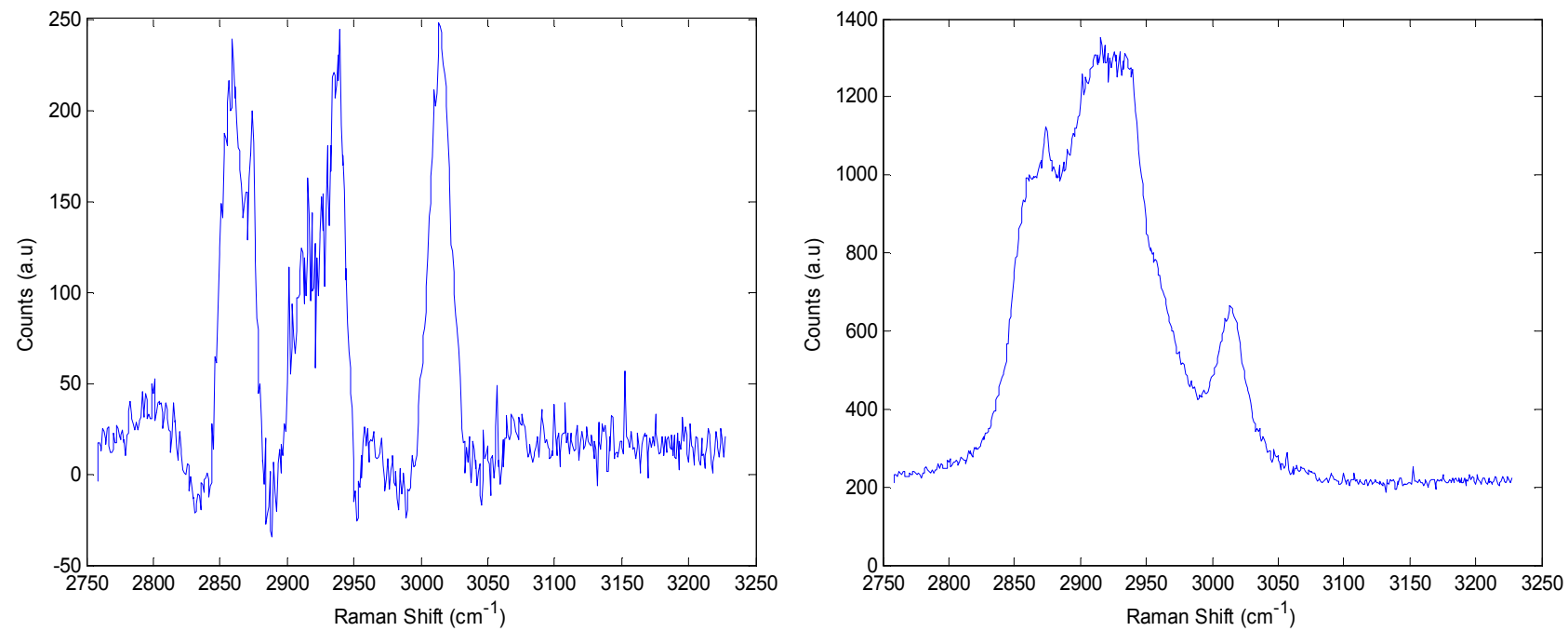

Figure 18. Gamma linolenic acid eli all-cis-6, 9, 12-octadecatrienoic acid (omega-6).
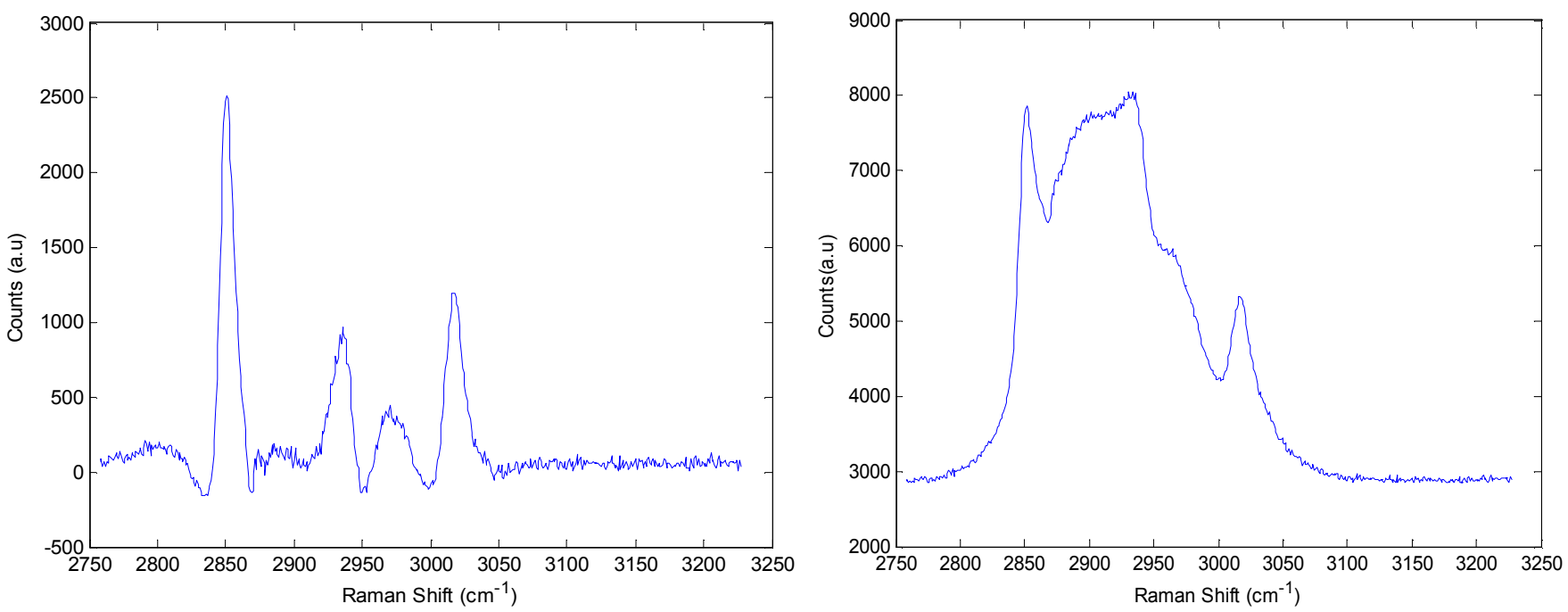

Figure 19. Sn-3-phosphatidylcholine-sn-2docosahexanoic acid-sn-1-stearic acid glycerol (22:6/18:0 PC).
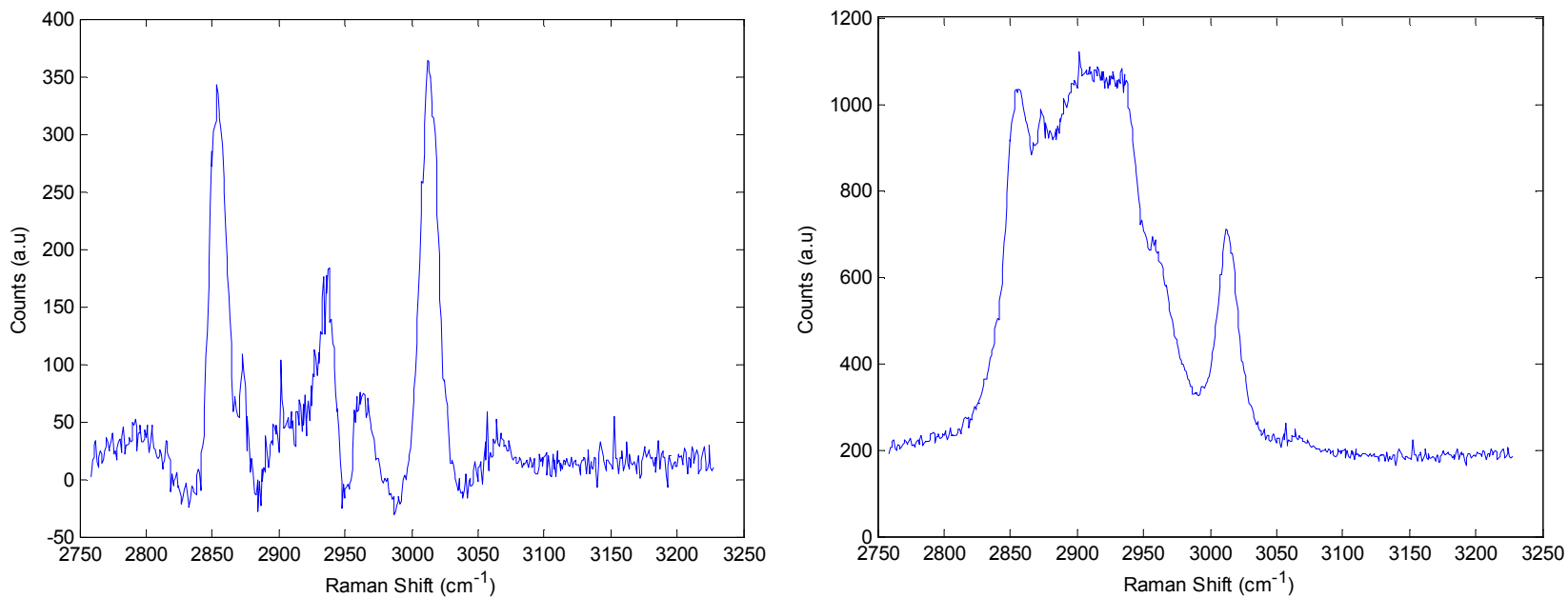

Figure 20. Linoleic/linoleic/alpha-linolenic acid. 

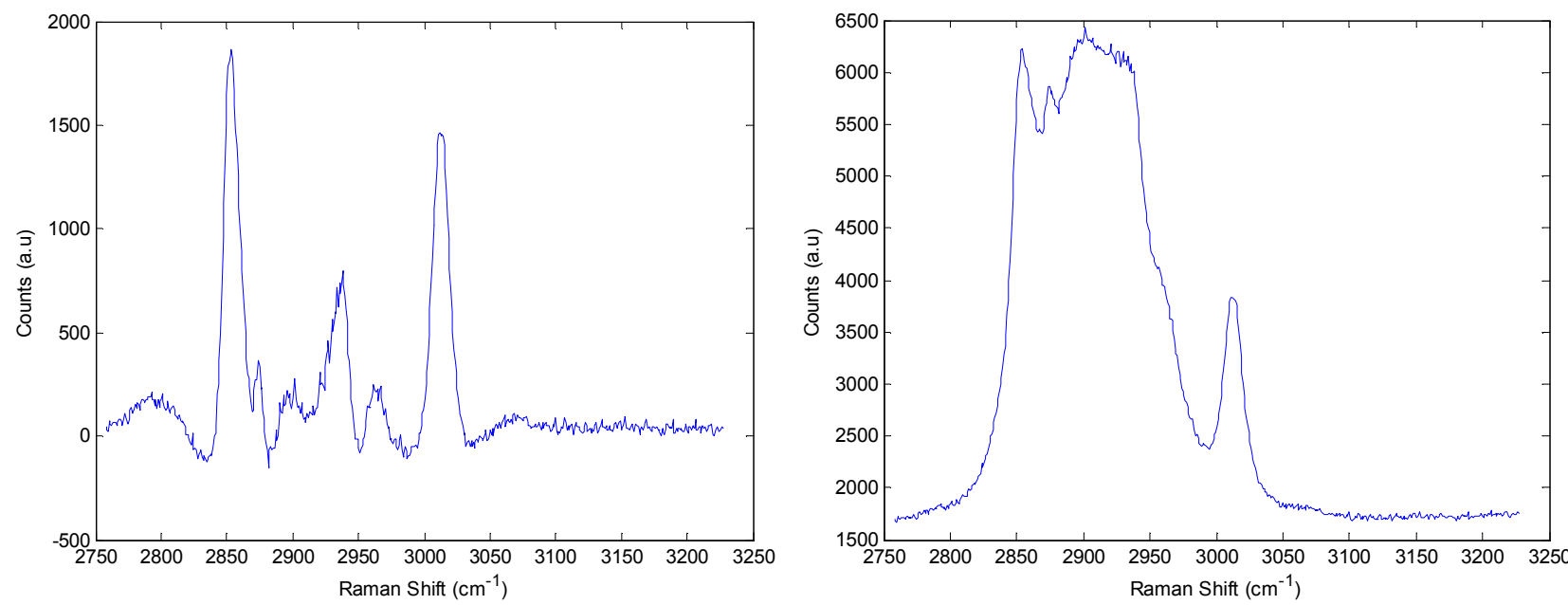

Figure 21. Sn-1 (sn-3)-linoleic acid Monoglyceride (18:2).

\subsubsection{Results of the SERS Measurement}
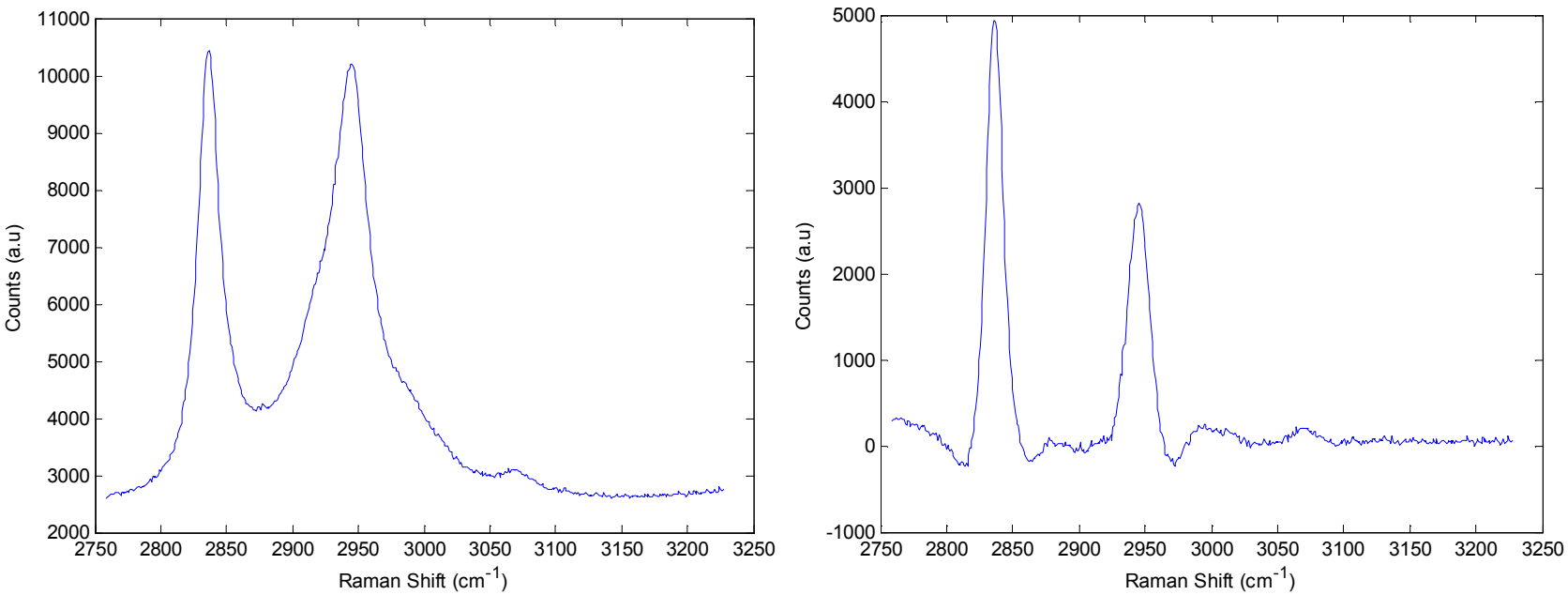

Figure 22. Palmitic acid eli hexadecanoic acid (Fatty acid 16:0).
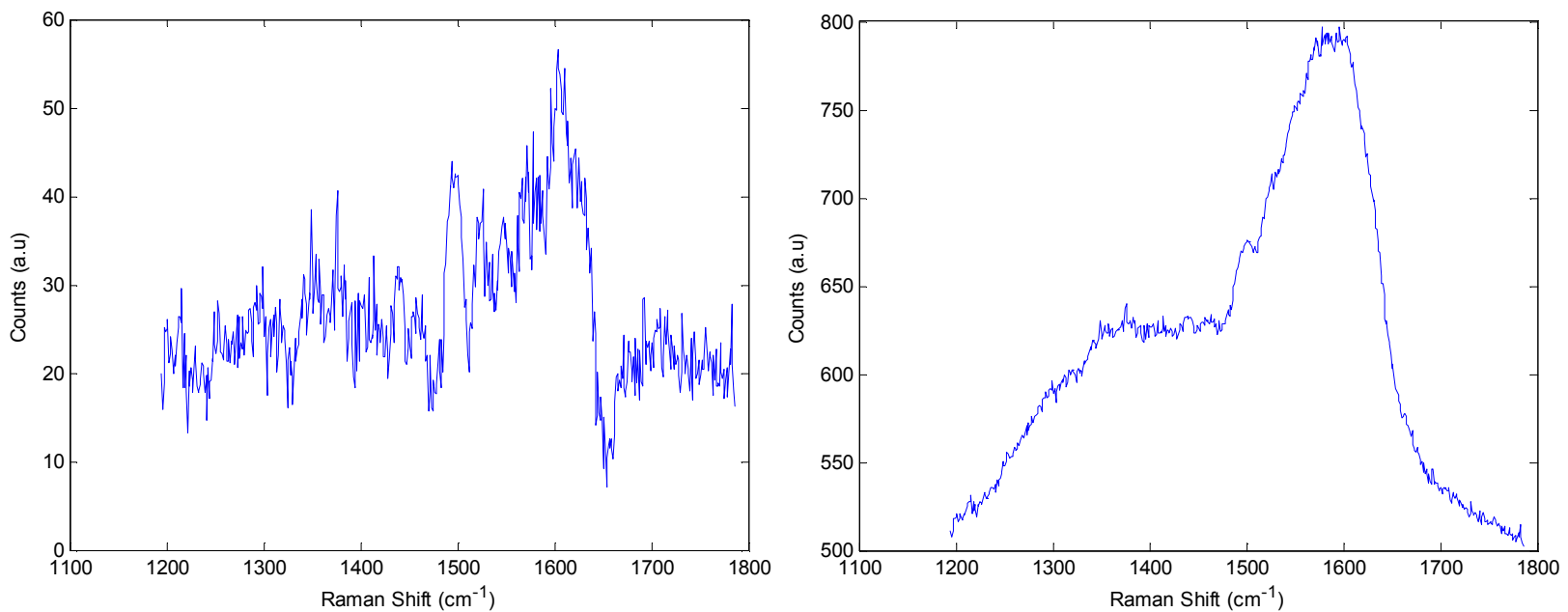

Figure 23. Stearic acid eli octadecanoic acid (Fatty acid 18:0). 

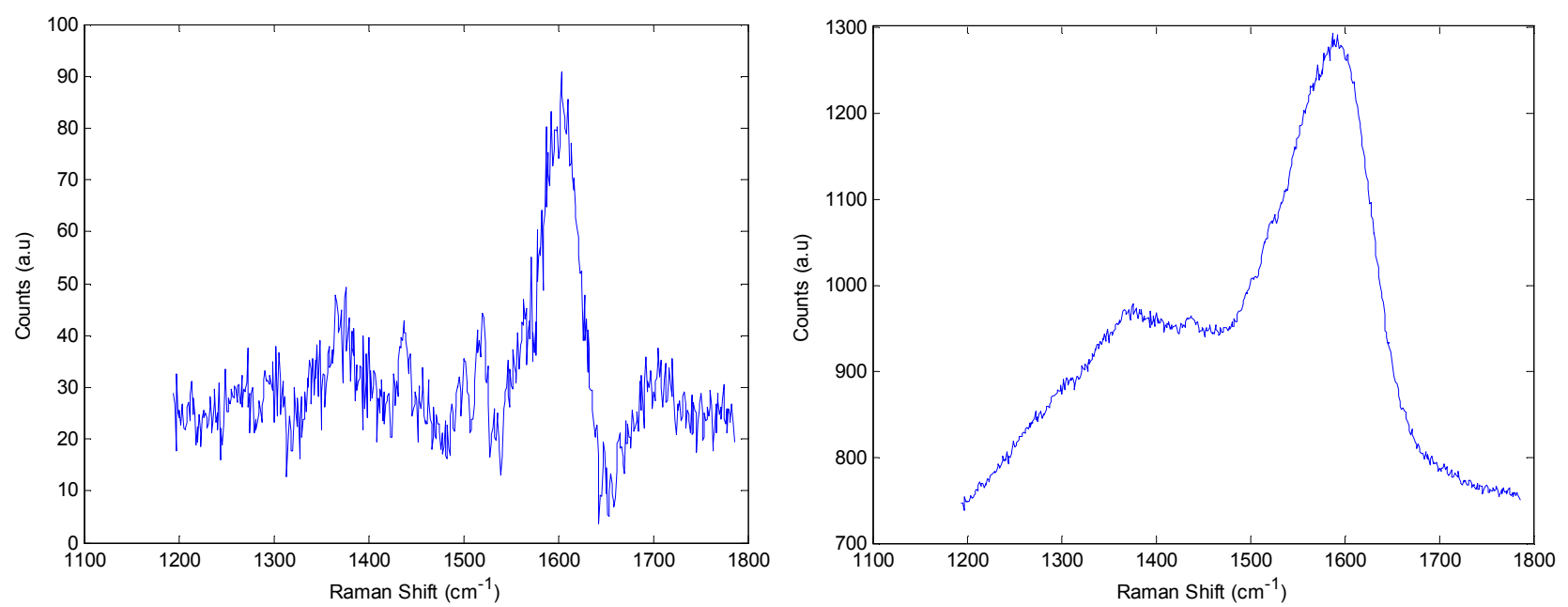

Figure 24. Oleic acid eli cis-9-octadecenoic acid (Fatty acid 18:1).
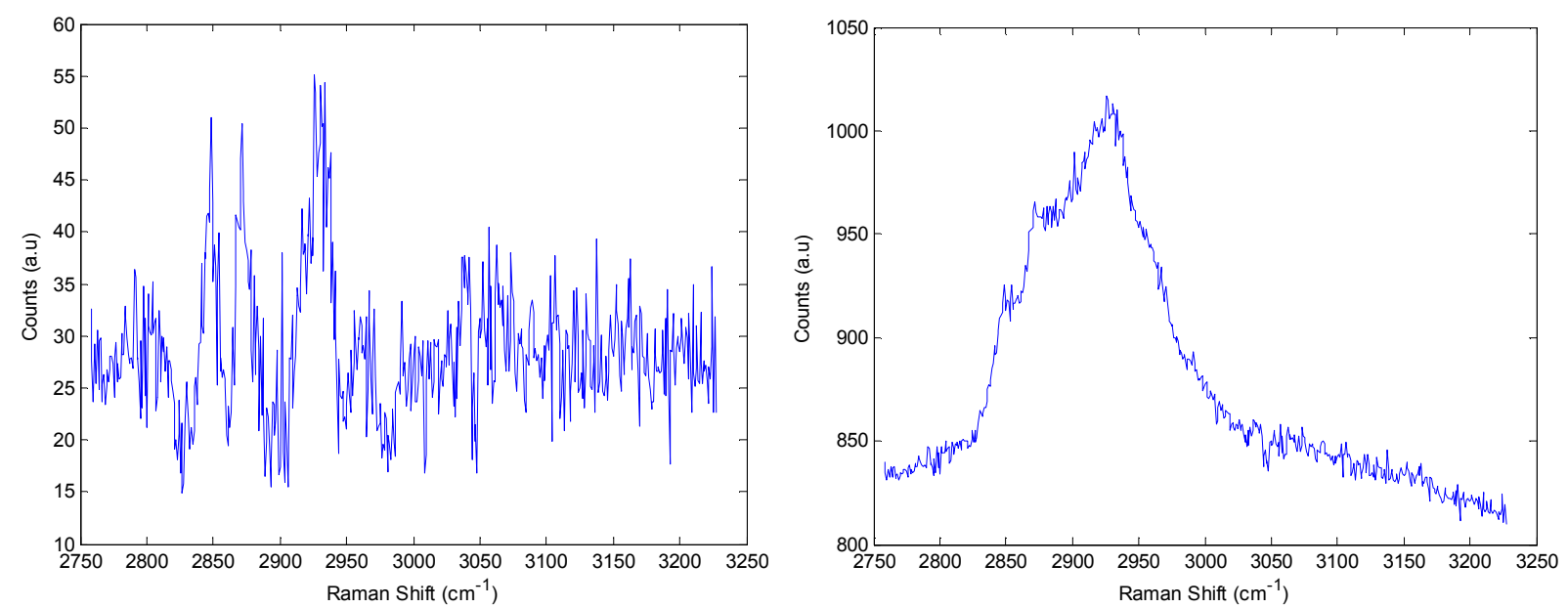

Figure 25. Sn-1 (sn-3)-stearic acid Monoglyceride (18:0).
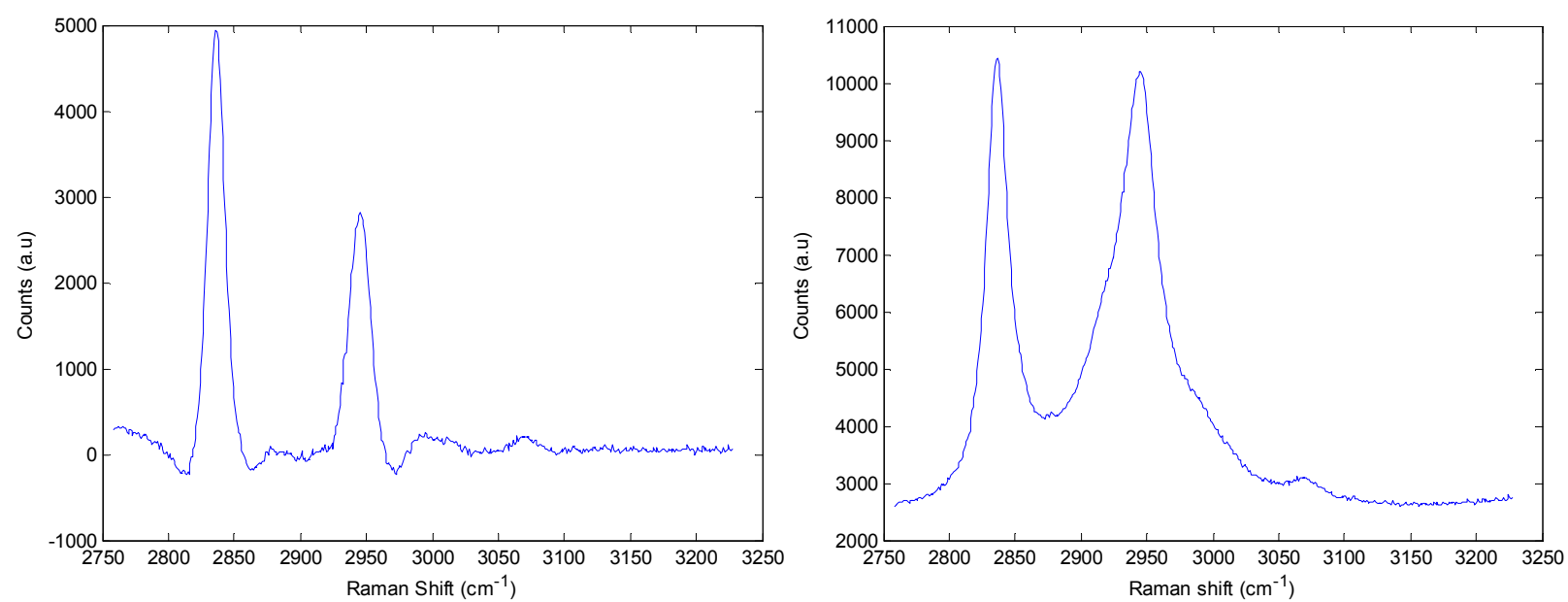

Figure 26. Palmitic acid eli hexadecanoic acid (Fatty acid 16:0)

\section{Discussion}

\subsection{Raman Results}

Stearic acid eli octadecanoic acid (Fatty acid 18:0)
Figure 12. Reviews Raman spectrum of 18 carbon atoms with zero double bonds of fatty acid which signify saturated fatty acid thus solid at room temperature due to bonds vibrations.

The peak at $28601 / \mathrm{cm}$ originates from the unit bond which is saturated fatty acid with high signal-to-noise ratio 
exhibited at the side of the bonds.

There is scissoring and twisting deformation (vibration) due to the zero double bond with reduced intensity.

Figure 12. Raman Spectrum of the longer shift clearly depicts saturated fatty acid with zero double bonds and 18 carbon atoms with a reduced signal-to-noise ratio due to variations in the zero double bonds.

Oleic acid eli cis -9-octadecanoic acid omega-9 (Fatty acid 18:1)

Figure 13. Reviews Raman spectrum of 18 carbon atoms with one double bond which signify unsaturated fatty acid.

The Raman peaks at $28501 / \mathrm{cm}$ and $29401 / \mathrm{cm}$ both signifies single bond whereas $30401 / \mathrm{cm}$ signify quasi double bonds which originates from the double bonds.

There is high signal-to-noise ratio due to the vibrations in the bonds with increased intensity.

There is also scissoring and twisting deformation due to the doubled bonds with increased intensity.

Figure 13. Raman spectrum of the longer shift shows reduced intensity with low signal-to-noise ratio.

The Raman peak at $30401 / \mathrm{cm}$ originates from double bond and can be used to identify unsaturated fatty acids from others.

sn-3 (sn-3)-stearic acid Monoglyceride (MAG 18:0)

Figure 14. Reviews Raman spectrum of Monoglyceride with 18 carbon atoms and no double bonds which signify saturated.

The Raman peak $2940 \quad 1 / \mathrm{cm}$ signify saturated Monoglyceride which originates from the double bond whereas the other peaks signifies single bonds with low signal-to noise ratio.

Figure14. Raman spectrum of the longer shift clearly depicts saturated Monoglyceride which originates from the double bonds with zero signal-to-noise ratio at a peak of $29401 / \mathrm{cm}$.

Palmitic acid eli hexadecanoic acid (Fatty acid 16:0)

Figure 15. Reviews Raman spectrum of a fatty acid with 16 carbon atoms with no double bonds thus saturated fatty acid.

The Raman peak at $29501 / \mathrm{cm}$ originates from the $\mathrm{C}=\mathrm{C}$ double bonds.

The double bonds can be used to predict saturated and unsaturated fatty acids from one another.

Figure 15. Raman spectrum of the longer shift depicts stretch with in phase aliphatic and vibrations due to $\mathrm{C}=\mathrm{C}$ double bonds.

Palmitoleic acid eli cis 9-hexadecenoic acid (Omega-7 Fatty acid 16:1)

Figure 16. Clearly depicts Raman Spectrum of fatty acid with length of 16 carbon atoms and one double bond thus signifying unsaturated fatty acid.

It clearly shows that, the peak at $30251 / \mathrm{cm}$ originates from the double bonds and can be used to describe and identify saturated and unsaturated fatty acids from one another.

Figure 16. The longer shift also depicts clearly double bonds with low-signal-to noise ratio and the double bonds are in -phase aliphatic at lower intensity which is amorphous due to vibrations in the double bonds.

Alpha-linolenic acid eli all-cis-9, 12, 15-octadecatrienoic acid (Omega-3 Ala Fatty Acid)

Figure 17. Reviews Raman spectrum of a fatty acid with higher signal-to-noise at both ends with increased intensity thus unsaturated fatty acid.

The peak at $30301 / \mathrm{cm}$ originates from the $(\mathrm{C}=\mathrm{C})$ double bond and can be used to identify unsaturated and saturated fatty acids from one another.

Figure 17. The spectrum of the longer shift depicts saturated fatty acid with low signal-to-noise ratio with in phase aliphatic, vibrations and stretch.

Gamma linolenic acid eli all-cis-6, 9, 12-octadecatrienoic acid (omega-6)

Figure 18. Reviews high-signal-to noise ratio at both ends of the bonds with increased intensity.

The peak at $30201 / \mathrm{cm}$ originates from the double bonds and can be used to identify unsaturated and saturated fatty acids from one another.

There is also partial deformation and stretching due to the double bonds.

Figure 18. Reviews spectrum of the longer shifts which signifies low signal-to-noise ratio due to stretching and vibrations in the double bonds thus unsaturated fatty acid.

Sn-3-phosphatidylcholine-sn-2docosahexanoic acid-sn-1stearic acid glycerol (22:6/18:0PC)

Figure 19. Reviews Raman spectrum of fatty acid with first 18 carbon atoms with no double bonds and the other 22 carbon atoms with six double bonds thus saturated fatty acid.

The spectrum peak at $30251 / \mathrm{cm}$ originates from the double bonds as well as the six double bonds which can be used to identify both saturated and unsaturated fatty acids from one another.

Figure 19. Signifies the longer shift which clearly depicts saturated fatty acid with low signal-to-noise ratio with slight stretching and vibration due to the double bonds.

Linoleic/linoleic/alpha-linolenic acid (LLAla TAG)

Figure 20. Reviews Raman spectrum of triglycerides which is unsaturated due to the double bond clearly observed at Raman peak $30251 / \mathrm{cm}$ with slightly high-to-signal ratio at reduced intensity.

There is also in-phase aliphatic and carbonyl stretch due to the $\mathrm{C}=\mathrm{C}$.

Figure 20. Signifies the longer shift with low signal-tonoise ratio due to stretching and slight vibrations in the bonds.

Sn-1 (sn-3)-linoleic acid Monoglyceride (MAG 18:2)

Figure 21. Signifies Raman spectrum of Monoglyceride with 18 carbon atoms and two double bonds thus unsaturated with low signal-to-noise ratio at reduced intensity.

The Raman peak at $30251 / \mathrm{cm}$ originates from the Monoglyceride double bonds which can be used to predict both saturated and unsaturated Monoglyceride from one another.

Figure 22. The longer shifts signify stretch deformation, scissoring, twisting and in-phase aliphatic due to the double 
bonds and other Raman peaks.

\subsection{SERS Results}

Palmitic acid eli hexadecanoic acid (Fatty acid 16:0)

Figure 22. The SERS measurement of the Fatty acid (16:0) with 16 carbon atoms and o double bonds thus signifying saturated fatty acid.

From figure 22 it clearly shows that, the peak at both 2825 $1 / \mathrm{cm}$ and $29501 / \mathrm{cm}$ exhibited some double bonds which could be used to identify both saturated, unsaturated, polysaturated and polyunsaturated fatty acid from one another'

Again there were higher intensity counts with low signalto-noise ratio.

Figure 22. The longer shifts also shows same features thus clearly peaks with good signal due to low concentration.

Stearic acid eli octadecanoic acid (Fatty acid 18:0)

Figure 23. Fatty acid with 18 length of carbon atoms and no double bonds thus saturated fatty acid.

There were no identification of peaks due to vibrations, solid nature of the molecules and out of phase aliphatic due to $\mathrm{C}-\mathrm{O}$ stretch with low signal-to-noise ratio.

Figure 23. The longer shift shows no double bonds due to twisting deformation at reduced intensity counts.

Oleic acid eli cis-9-octadecenoic acid (Fatty acid 18:1)

Figure 24. Raman spectrum with 18 length of carbon atoms with one double bond thus signifying unsaturated fatty acid.

There were unseen series of sub-peaks generated due to the spreading of the analyte at a diluted concentration (higher concentration) but the peak at $16501 / \mathrm{cm}$ originates from the double bond which could be used to predict saturated and unsaturated fatty acids from each other at a reduced intensity counts.

Figure 24. The longer shift clearly depicts the fingerprint which shows clearly 18 carbon atoms with one double bond at no oxidation.

Sn-1 (sn-3)-stearic acid Monoglyceride (18:0)

Figure 25. Monoglyceride with 18 carbon atoms and no double bonds thus signifying saturated.

This originates from the solid nature of the molecules at a higher concentration.

Figure 25. The longer shift signifies higher signal-to-noise ratio due to twisting deformation at $\mathrm{C}-\mathrm{O}$ and out-of-phase aliphatic.

Palmitic acid eli hexadecanoic acid (Fatty acid 16:0)

Figure 26. 18 carbon atoms and no double bonds thus saturated fatty acid.

The peak at $29501 / \mathrm{cm}$ originates from a double bond with low concentration of analyte at low-signal-to noise ratio, low intensity counts which can be used to identify saturated, polysaturated, unsaturated, polyunsaturated fatty acids from one another.

Figure 26. The longer shift clearly shows double bond with low concentration, high intensity counts and amorphous nature of the analyte.

\section{Conclusions}

The goal of this thesis was to use Raman and Surfaceenhanced Raman spectroscopy (SERS) to study and identify the optical and molecular structure of fatty acids and lipids where the peaks originated from.

This study reveals that, both the Raman spectroscopy and SERS analysis of the fatty acids and lipids clearly depicts that most of the peaks obtained originated from the double bonds where it was used to identify saturated and unsaturated fatty acids and lipids from one another.

I observed that, SERS normally occurs at a very low concentrations whereas the Raman measurement occurs at both higher and quasi- lower concentrations. I also observed that, the main difference between Raman and SERS was that, the first has weaker scattering, no sample preparation, allowing in-situ, non-invasive and the incident radiation occurs at a percentage of $\left(10^{-6} \%\right)$ whereas the latter it reveals that, the degree of the enhancement depends on the shape, size, surface and nature of the analyte.

I observed that, the $514 \mathrm{~nm}$ excitation used is very negligible to correctly and efficiently execute the surface Plasmon.

For clearly peaks to be seen in the latter, it was observed that, the analyte and laser excitation has to be positioned either vertically or elliptically polarized orthogonally to the surface.

Finally it was found that only (2-3\%) of the SERS could be seen in the fatty acids and glycerides.

\section{Appendix}

Raman and SERS peaks simulations with matlab codes function example_code

ReadRamanSPCfiles

title $1=$ ans.Read.Name

$\%$ for loop for stacking all the spectra in one matrix for $j=1: 16 \%$ number of measured spectra

xaxis=[ans.Read.spectra(j).xaxis]'; \% Raman shift region (x-axis) of each spectra

Data $=[$ ans.Read.spectra(j).data]'; \% Raman intensity (yaxis) of each spectra

Baseline $=[$ ans.Read.spectra(j).baseline]'; \% baseline defined in ReadRamanSPCfiles function

Data_cor=Data;

if $j==1$

Data_all=Data_cor;

Data_all_base=Data_cor-Baseline;

else

Data_all=[Data_all Data_cor]; \% Matrix containing the raw data 
Data_all_base=[Data_all_base Data_cor-Baseline]; \% Matrix containing baseline corrected data

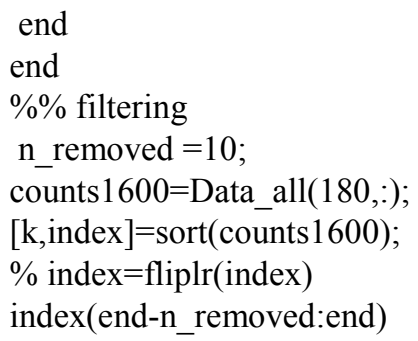

Data_all(:,[index $($ end-n_removed:end $)])=[] ; \quad \%$ remove large $1 \overline{6} 00$

Data_all_base $(:,[$ index $($ end-n_removed:end $)])=[]$;

Data_all_average $=$ mean(Data_all,2); \% averaging of the raw spectra

Data_all_base_average $=$ mean $($ Data_all_base, 2$)$;

figure

plot(xaxis,Data_all_average)\% plotting the raw spectra as

a function of Raman shift

figure

plot(xaxis,Data_all_base_average)

xlabel('Raman Shift $\left.\left(\mathrm{cm}^{\wedge}{ }^{-} \wedge 1\right) '\right)$

ylabel('Counts (a.u)')

\section{$\% \%$ saving}

tothefile $=[$ xaxis Data_all $]$

cd E:ISERS_codes $\backslash$ average

save(title1,'-ascii', '-tabs','tothefile')

cd E:ISERS_codes $\backslash$

$\% \%$

end

\section{References}

[1] ATA Scientific. (2020). Spectrometry And Spectroscopy: What's The Difference? https://www.atascientific.com.au/spectrometry/

[2] Balčytis, A., Nishijima, Y., Krishnamoorthy, S., Kuchmizhak, A., Stoddart, P. R., Petruškevičius, R., \& Juodkazis, S. (2018). From fundamental toward applied SERS: shared principles and divergent approaches. Advanced Optical Materials, 6 (16), 1800292.

[3] Beyssac, O. (2020). New trends in Raman spectroscopy: from high-resolution geochemistry to planetary exploration. Elements: An International Magazine of Mineralogy, Geochemistry, and Petrology, 16 (2), 117-122.
[4] Bwtek, (2015). Spectrometer. http://bwtek.com/spectrometerpart-5-spectral -resolution/ (valid 2015).

[5] Palmer, C. Diffraction Grating Handbook, $2^{\text {nd }}$ ed. (Wiley, New York, 1997). Camden, J. P., et al., Journal Am Chem Soc (2008).

[6] Caprara, D., Ripanti, F., Capocefalo, A., Ceccarini, M., Petrillo, C., \& Postorino, P. (2021). Exploiting SERS sensitivity to monitor DNA aggregation properties. International Journal of Biological Macromolecules, 170, 8893.

[7] Chen, X., Gu, H., Shen, G., Dong, X., \& Kang, J. (2010). Spectroscopic study of surface enhanced Raman scattering of caffeine on borohydride-reduced silver colloids. Journal of Molecular Structure, 975 (1-3), 63-68.

[8] E. Amankwa, (2016). Characterization of Designed and Constructed Optical Systems, Texila International Journal of Academic Research.

[9] Fleischman, M., et al., (1974) Chem Phys Lett.

[10] Jaculbia, R. B., Imada, H., Miwa, K., Iwasa, T., Takenaka, M., Yang, B.,... \& Kim, Y. (2020). Single-molecule resonance Raman effect in a plasmonic nanocavity. Nature nanotechnology, 15 (2), 105-110.

[11] Jeanmaire, D. L, and Van Duyne, R. P., (1977). Journal Electro anal Chem.

[12] Langer, J., Jimenez de Aberasturi, D., Aizpurua, J., AlvarezPuebla, R. A., Auguié, B., Baumberg, J. J.,... \& Liz-Marzán, L. M. (2019). Present and future of surface-enhanced Raman scattering. ACS nano, 14 (1), 28-117.

[13] Liu, F., Song, B., Su, G., Liang, O., Zhan, P., Wang, H.,... \& Wang, Z. (2018). Sculpting extreme electromagnetic field enhancement in free space for molecule sensing. Small, 14 (33), 1801146.

[14] Martín-Yerga, D., Pérez-Junquera, A., González-García, M. B., Perales-Rondon, J. V., Heras, A., Colina, A.,... \& FanjulBolado, P. (2018). Quantitative Raman spectroelectrochemistry using silver screen-printed electrodes. Electrochimica Acta, 264, 183-190.

[15] Moore, T. J., Moody, A. S., Payne, T. D., Sarabia, G. M., Daniel, A. R., \& Sharma, B. (2018). In vitro and in vivo SERS biosensing for disease diagnosis. Biosensors, 8 (2), 46.

[16] Nicole K., Robert D. Simoni and Robert L. Hill, JBC Historical Perspective: Lipid Biochemistry (2010), The American Society for Biochemistry and Molecular Biology, Inc. printed in the USA. http:www.oceanoptics.com/products/benchopticsge.asp.

[17] P. Y. Bruce, Organic Chemistry (4 ${ }^{\text {th }}$ ed, 2006).

[18] Platt, U. \& Stutz, J., (2008). Differential Optical Absorption Spectroscopy. Springer Berlin Heidelberg.

[19] Silva, I. (2020). Raman Spectroscopy. Between Making And Knowing: Tools In The History Of Materials Research, 435.

[20] Vahimaa, P., Nuutinen, T., Mtikainen, A., Dniel, S., Kwarkye, K., Amankwa, E., \& Andoh, S. (2016). Surface-Enhanced Raman Spectroscopy http://www.uef.fi/en/web/photonics/sers.
(SERS). 
[21] Vitha, M. F. (2018). Spectroscopy: Principles and Instrumentation. John Wiley \& Sons.
[22] Vitlina, R. Z. E., Magarill, L. I., \& Chaplik, A. V. (2018). Raman scattering by plasma oscillations in quantum rings. JETP Letters, 108 (5), 292-295. 